\title{
Significance of podoplanin expression in cancer- associated fibroblasts: A comprehensive review
}

\author{
BARTOSZ PULA $^{1,2}$, WOJCIECH WITKIEWICZ ${ }^{1}$, PIOTR DZIEGIEL ${ }^{1-3}$ and MARZENA PODHORSKA-OKOLOW ${ }^{1,2}$ \\ ${ }^{1}$ Regional Specialist Hospital, Research and Development Center, 51-124 Wroclaw; ${ }^{2}$ Department of Histology \\ and Embryology, Medical University, 50-368 Wroclaw; ${ }^{3}$ Department of Physiotherapy, \\ Wroclaw University School of Physical Education, 51-612 Wroclaw, Poland
}

Received January 17, 2013; Accepted March 14, 2013

DOI: $10.3892 /$ ijo.2013.1887

\begin{abstract}
Cancer-associated fibroblasts (CAFs) are well-known to be part of the tumor microenvironment. This heterogeneous population of cells of the tumor microenvironment via secretion of various growth factors and cytokines was shown to contribute to increased cancer cell proliferation rate, migration, invasiveness and other key processes such as angiogenesis and lymphangiogenesis. Recent studies identified podoplanin as a marker of CAFs in various malignancies and its expression in these cells was shown to influence cancer progression. In some studies it yielded a prognostic impact on patient survival which was strongly dependent on the entity of the tumor. This review summarizes recent findings concerning the biology of podoplanin in cancer progression with particular emphasis on its expression in CAFs.
\end{abstract}

\section{Contents}

1. Introduction

2. Podoplanin discovery, structure and expression regulation

3. Podoplanin and lymphatic vessel development

4. Podoplanin and tumor metastasis facilitated by thrombus formation

5. Podoplanin and cancer cell migration, invasion and progression

6. Podoplanin expression in cells of normal and cancerous stroma

7. Conclusion

\section{Introduction}

As early as 1863 Rudolf Virchow hypothesised that cancer cell growth may be stimulated by surrounding stromal cells

Correspondence to: Dr Marzena Podhorska-Okolow, Department of Histology and Embryology, Medical University of Wroclaw, Chalubinskiego 6a, 50-368 Wroclaw, Poland

E-mail: marzenna.podhorska-okolow@umed.wroc.pl

Key words: podoplanin, cancer-associated fibroblasts, stroma and that the neoplastic process begins at the site of chronic inflammation, since leukocytes were seen in the stroma of neoplastic tissues (1). This hypothesis is actually somewhat true since recent research has shown that inflammatory cells may contribute to the development and progression of various malignancies $(2,3)$. Besides inflammatory cells, in the majority of various tumors fibroblasts are seen (4). Under normal conditions, fibroblasts synthesize the components and regulate the deposition of the extracellular matrix (ECM), epithelial differentiation and inflammation (5-8). Fibroblasts are also key in the process of wound healing and fibrosis during which they exert different properties than those of normal fibroblasts $(6,9)$. Their phenotype also changes, resulting in increased proliferation, motility and synthesis of various growth factors, referred to as 'activation' (4).

Cancers are often called 'wounds that do not heal'. Recent research has shown that fibroblasts of cancerous stroma, the so-called cancer-associated fibroblasts (CAFs), exert an 'activated phenotype' in the majority of the studied malignancies $(4,7,10,11)$. There is evidence which suggests that CAFs are characterized by a distinct gene expression profile, and that their properties differ from those of normal fibroblasts (12). Furthermore, CAFs may be incorporated into the tumor environment via different pathological mechanisms $(4,7,13)$. Transformation of normal fibroblasts under the regulation of cancer cells has been proposed. However, it is becoming apparent that the recruitment of bone marrow derived stem cells, trans-differentiation of cancer (endothelial-mesenchymal transition; EMT) or endothelial cells (endothelial-mesenchymal transition; EndMT) may also very significantly contribute to this process $(4,7,13,14)$. CAFs of invasive breast cancers were shown to augment tumor growth and induce angiogenesis via recruitment of endothelial cells due to secretion of high levels of stromal-derived growth factor (SDF-1) (15). CAFs were also shown to promote tumor growth by secreting ECM-degrading proteases (MMPs), hepatocyte growth factor (HGF) or connective tissue growth factor (CTGF) (16-22). Moreover, it was found that CAFs contribute to tumor progression via recruitment of inflammatory cells into the tumor environment (23). CAFs may also modulate cancer stem cell phenotype, as was recently described in colorectal carcinoma $(24,25)$.

Fibroblasts are a heterogeneous group of cells. Their phenotype is strongly dependent on the tissue origin and topography. 
As a result, tissue specific markers of fibroblast activation are of particular importance (26). The $\alpha$-smooth muscle actin ( $\alpha$ SMA) is regarded as a marker of activated CAFs in most of the studies $(4,6,27)$. Besides $\alpha$ SMA, vimentin, desmin, fibroblast-specific protein 1 (FSP1) or fibroblast-activation protein are also used to identify and characterize CAFs in human malignancies (27-33). There is also evidence that different subsets of CAFs are present in the tumor stroma and differ in their properties (34). Recent findings have identified podoplanin as a potentially new marker of CAFs in various malignancies, giving new insight into the biology of cancers and the tumor microenvironment $(27,28,35-37)$.

\section{Podoplanin discovery, structure and expression regulation}

Podoplanin was first discovered in 1990, when its mRNA was found in the murine osteoblastic cell line (MC3T3-E1) and ras-transformed cells (38). In 1996, Wetterwald et al raised an antibody which could detect podoplanin (designated as E11 antigen due to the antibody clone) in rat osteoblasts, preosteocytes and osteocytes (39). Podoplanin (E11) positive cells were also identified as alveolar type 1 cells in lung, endothelial cells of choroid plexus and lymphatic endothelial cells (LECs) (39). Podoplanin was named for the above mentioned protein when Breiteneder-Geleff et al, discovered for the first time its expression on rat podocytes (40). In 1999 podoplanin was accepted as a novel marker of lymphatic endothelial cells (Fig. 1) (41,42).

The most popular synonym for podoplanin is D2-40, stemming from the name of the antibody clone which is widely used for its detection in paraffin-embedded tissues $(27,43)$. Podoplanin, is also known as gp36, gp38, canine gp40, T1 $\alpha$, PA2.26, Aggrus, OTS-8 or M2A oncofetal antigen (38,44-50).

Podoplanin is a 162 -amino acid transmembrane sialoglycoprotein belonging to type-1 transmembrane sialomucin-like glycoproteins (51). Its mass varies from 38 to $50 \mathrm{kDa}$ due to the extent of the sialilation of its extracellular domain with numerous serine and threonine amino acid residues $(42,52,53)$. Podoplanin consists of a small transmembrane domain and an intracellular domain. The latter interacts with protein kinase $\mathrm{C}$ (PKC) and proteins of the ERM (ezrin, radixin, meosin) family, which were shown to influence cancer cell motility and invasive potential $(51,54)$. The podoplanin gene consists of $34.4 \mathrm{~kb}$ and 8 exons. To date 2 isoforms of podoplanin have been discovered utilizing northern blotting. They probably resemble a product of alternative splicing, but the biological significance of this finding remains to be clarified $(53,55)$. Its expression is regulated on the level of transcription (numerous initiation sites, alternative splicing, polyadenylation) and on the post-translational level due to podoplanin calpain-mediated proteolysis $(53,56)$. Although the exact mechanism of its expression regulation remains unknown, numerous factors ranging from micro-RNA to signalling factors have been seen (42,57-60).

\section{Podoplanin and lymphatic vessel development}

As mentioned above, podoplanin is regarded as a marker of lymphatic vessel endothelium $(41,42)$. Podoplanin-positive cell structures were shown to be simultaneously positive for Prox1 and VEGFR-3 (61). It seems that VEGF-C upregulates podoplanin expression via Proxl, which is a master regulator gene of lymphatic vessel development facilitating LECs differentiation from lymphatic progenitor cells in embryonic veins $(61,62)$. Podoplanin expression was also shown to be upregulated by IL-3 in dermal LECs (63).

The important role of podoplanin in lymphatic vessel development has been made clear by experiments performed with podoplanin-deficient mice which die at birth from respiratory failure caused by abnormal alveolar sac development due to the absence of podoplanin expression in alveolar type I cells $(48,64)$. In these mice there were serious lymphatic abnormalities including abnormal lymphatic vessel patterning, lymph transport and lymphedema combined with lymphatic vessel dilatation (48). Although no connections between the blood and lymphatic vessels were reported in the above mentioned studies (the so-called 'separation phenotype'), Fu et al showed that mice deficient in endothelial O-glycans, from the targeting of T-synthase required for their production, possess a 'non-separation' phenotype with numerous misconnections between both vessel types (65). Mice lacking the T-synthase were also characterized by impaired podoplanin expression on endothelial cells. As a result, it was hypothesized that the abnormal connections between the blood and lymphatic vessels occurred due to the lack of its expression (65). Recently Uhrin et al showed that podoplanin platelet-aggregating properties are crucial for proper lymphatic vessel development. Thrombi are formed at sites of podoplanin-positive lymph sacs separating them from cardinal veins during mouse embryonic development, thereby enabling proper separation of blood and newly formed lymphatic vessels (66).

\section{Podoplanin and tumor metastasis facilitated by thrombus formation}

Recent findings have led to new discoveries which help clarify the functions of particular podoplanin domains. The extracellular domain of podoplanin consists of the EDxxVTPG segment (PLAG - platelet aggregating domain), which is responsible for platelet aggregation (51). C-type lectin-like receptor-2 (CLEC-2) was discovered as the first podoplanin receptor on human platelets responsible for platelet aggregating properties of podoplanin $(67,68)$. Recombination of CLEC-2 or mutations in the threonine residues of PLAG result in abolished platelet aggregation by podoplanin $(47,67)$. Podoplanin interaction with platelets was also shown to be inhibited by antibodies directed against CLEC-2, which may be significant in anticancer therapies $(68,69)$. It was shown that tumor cells induce platelet aggregation which protects cancer cells from sheer stress and host immunological defence (70). This phenomenon may result in increased tumor growth and enhanced metastatic potential of the tumors $(47,71,72)$. Using animal models it was shown in vivo that antibodies targeting the interaction of the PLAG domain and CLEC-2 may suppress tumor metastasis $(68,73)$. The blockade of CLEC-2 may be of potential importance since diminished functionality of this receptor results in decreased thrombus formation with a non-significant clinical rise in bleeding time (72,74-76). However, caution should be taken when using antibodies targeting podoplanin function 


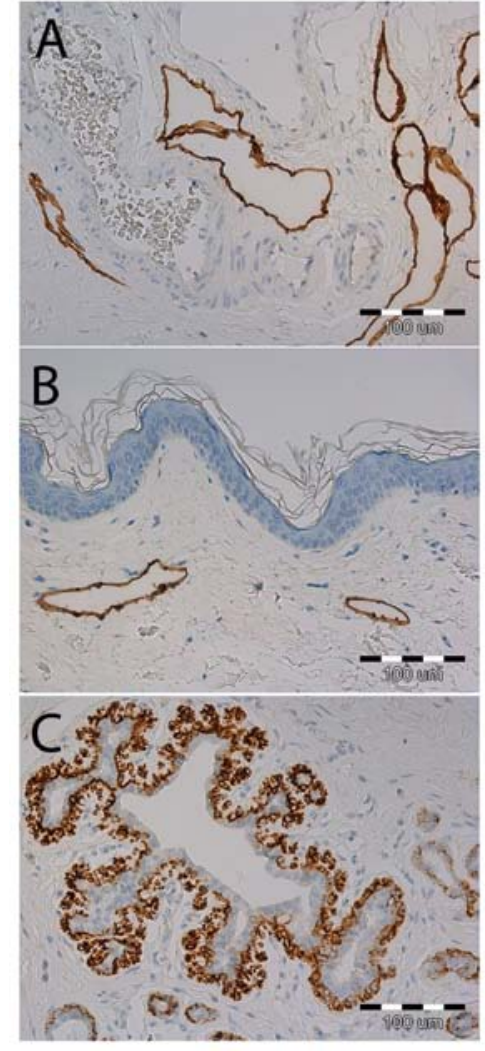

Figure 1. A membrane-cytoplasmic expression pattern of podoplanin in the lymphatic vessel endothelium was utilized to detect lymphatic vessels in various tissues, as none of its expression is noted in the blood vessel endothelium (A and B). Podoplanin expression in myoepithelial cells surrounding breast duct cells $(\mathrm{C})$. None of its expression is noted in the normal, non-transformed stroma. because podoplanin-Fc transgenically expressed in mouse skin resulted in lethal disseminated intravascular coagulation (77).

\section{Podoplanin and cancer cell migration, invasion and progression}

Several studies and reviews have already shown that podoplanin affects the migration of normal and cancer cells $(42,52,59,78)$. Cancer cells may migrate in two distinctive patterns. The most often studied theory is based on the single cell migration model which is often connected to the EMT phenomenon. In this process, cancer cells lose their epithelial phenotype e.g., loss of E-cadherin, and acquire a mesenchymal phenotype which is characterized by expression increase of the mesenchymal markers N-cadherin, $\alpha$ SMA or FSP-1. This is often regarded as the cadherin switch (52). This process results in the increase of migratory and invasive potential, and cancer cell resistance towards apoptotic stimuli (79). Although this process is considered crucial in cancer cell dissemination, it is rarely observed in paraffin sections of embedded cancer tissues. On the contrary, large cancer cell bulks invading the neighboring tissues are most frequently observed in cancerous tissues. In this model, known as 'collective cell migration', cancer cells maintain the expression of epithelial markers (52). Experimentation has indicated that podoplanin may mediate cancer cell migration in both hypothetical invasion models.

Wicki et al in the Rip1Tag 2 model of pancreatic $\beta$-cell carcinogenesis have shown that transgenic expression of podoplanin in pancreatic $\beta$-cells led to the formation of carcinomas in the absence of EMT, since the cancer cells

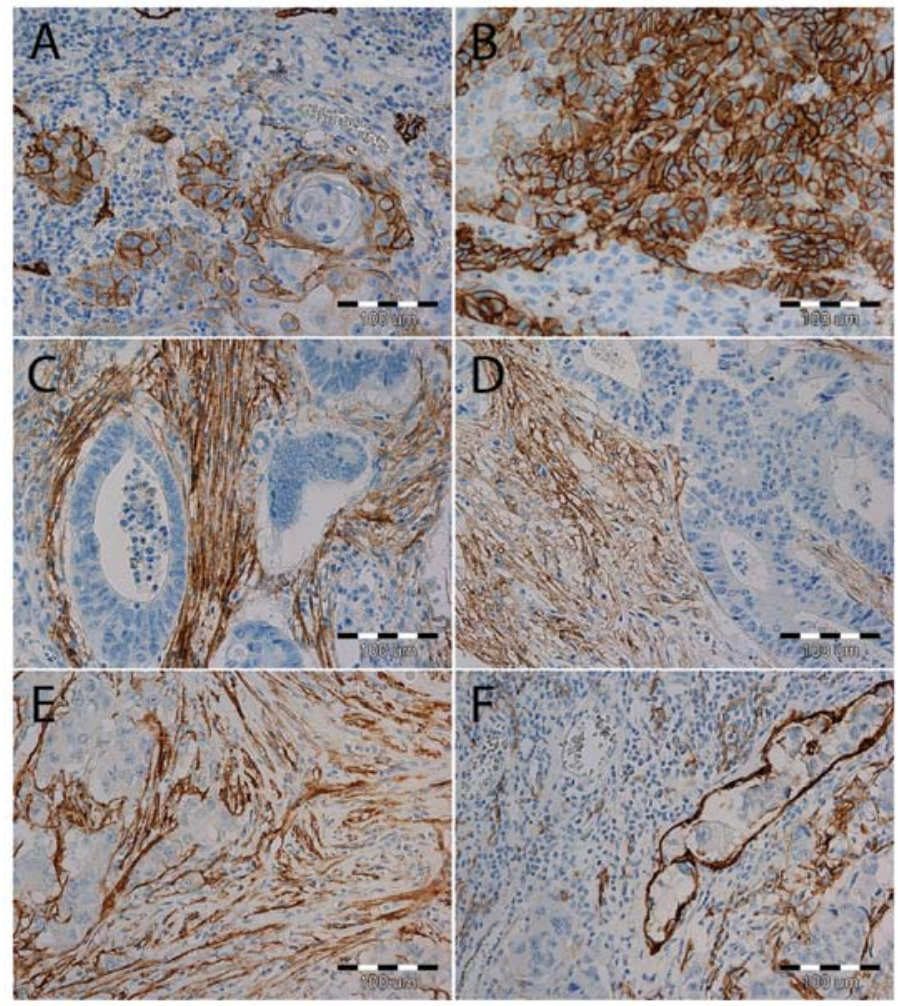

Figure 2. Podoplanin membrane staining of squamous cancer cells of skin (A) and lung (B) cancers. On the contrary no staining is visible in the adenocarcinoma of the lung (C), colon (D) and invasive ductal breast carcinoma (E and F). In these tumors, podoplanin expression is mainly noted in the CAFs of tumour stroma (C-E) and LECs (F). 
invaded the surrounding tissues in a collective pattern (59). In this experimental model mice develop adenomas and carcinomas of $\beta$-cells in pancreatic Langerhans islets due to the expression of simian virus large $\mathrm{T}$ antigen under the control of rat insulin promotor $(52,59)$. Moreover, podoplanin colocalized with E-cadherin on the invading tumor edge in various cancers of tumor origin $(52,59)$. Neo-expression of podoplanin in MCF-7 (breast cancer cell line) supported this observation since no cadherin switch occurred in these cells. Nevertheless, the cells were characterized by increased migratory and invasive potential (59). Under the influence of transforming growth factor $\beta$ (TGF $\beta$ ), epidermal growth factor (EGF) and hepatocyte growth factor (HGF), an increase in podoplanin expression could be noted in the podoplanin transfected MCF-7 cells (59). It is noteworthy that podoplanin is rarely expressed in breast cancer cell lines and tissues, not even in the most invasive and metastasizing cell lines showing expression of mesenchymal markers (MDA-MB-231 and MDA-MB-231. BO2) $(27,36,53)$.

Podoplanin neo-expression in Madin-Darby canine kidney (MDCK) cells resulted in EMT. The cells acquired migratory features dependent on the interaction of podoplanin endodomain with ezrin and radixin, proteins of the ERM complex of cell membrane. Podoplanin binding to these proteins resulted in activation of RhoA small G protein (54). Moreover, induction of podoplanin expression in epidermal MCA3D keratinocytes (3D2.26) induced cell surface extensions, had increased motility, a loss of cortical actin and destabilization of adherens junctions. In addition, these cells also had a loss of keratin-14 and an increase of vimentin and keratin-14 expression. Upon injection into athymic mice, 3D2.26 cells formed undifferentiated tumors with aberrant E-cadherin expression and formation of tumor metastases in regional lymph nodes. This highlights the malignant transformation of these cells (80). Podoplanin was also shown to be upregulated in human epideral keratinocytes by TGF $\beta$, IFN $\gamma$, IL- 6 and IL-22, which partially confirms the findings in the MCF-7 cells (57).

Podoplanin was also identified in raft platforms, structures crucial for cell signaling (81). It seems that the short GXXXG motif of the podoplanin transmembrane domain is crucial for its association with detergent-resistant membranes (DRMs), ERM phosphorylation or induction of EMT and cell migration (81). MDCK cells with mutation in the GXXXG motif of podoplanin transmembrane domain were characterized by their impaired dimerization and lacked localization in the lipid raft platforms resulting in the absence of EMT (81). Apparently podoplanin inclusion in the raft platforms is crucial for its interaction with the actin cytoskeleton and activation of the RhoA/ROCK pathway. A recent study showed that podoplanin interacts with CD44 independently of ERM proteins in cell membrane protrusions which therefore ensures directional cell migration (82). CD44 is an ERM binding protein. Its interaction with actin cytoskeleton is also dependent on its inclusion in the lipid rafts (83). It therefore appears that podoplanin regulates the RhoA/ROCK activity via its inclusion (81-83).

Although it is clear that podoplanin mediates migration and invasiveness of cancer cells, the biology and interactions of this protein seem to be strongly dependent on the cell type. Martin-Villar et al and Fernandez-Munoz et al demonstrated that podoplanin expression in MDCK cells led to induced activity of RhoA, whereas Wicki et al, demonstrated a decrease of RhoA, Racl and cdc42 activity upon podoplanin introduction into the MCF-7 cells $(54,59,81)$.

It has also been shown, that the Src kinase stimulates podoplanin expression in the homozygous null gap junction Cx43 (connexin 43) knock-out brain cells by phosphorylating the focal adhesion adaptor protein Cas (Crk associated substrate). This led to induced cell migration (84). Interaction of the Src kinase with Cas was shown to promote anchorage-independent growth and migration of cancer cells and these effects may be mediated by induction of podoplanin expression (84). In addition, podoplanin expression was found to be decreased by contact normalization, a process that forces the cells to maintain normal non-transformed cell phenotype, therefore preventing their malignant transformation $(84,85)$.

A recent study of Acton et al identified podoplanin as an inducer of dendritic cell (DC) migration via interaction with its receptor, the CLEC-2 protein (86). Binding of podoplanin expressed on fibroblastic reticular cells (FRCs) and LECs with CLEC-2 on DCs resulted in increased migratory potential of the latter. This mechanism might also be responsible for the migration of cancer cells along the lymphatic networks since the neoexpression of CLEC-2 in a human A375 melanoma cell line resulted in protrusion formation upon contact of the CLEC-2 overexpressing A375 melanoma cell line with podoplanin expressing FRCs (86).

Diversity of podoplanin biology is also apparent in different tumor types. Podoplanin expression in breast cancer cell lines augmented their metastatic potential and dissemination through villin-1 dependent induction of lymphangiogenesis (87). On the contrary, forced expression in the lung squamoid EBC-1 cancer cells attenuated prolymphangiogenic and metastatic potential by reducing the expression of VEGF-C in these cells via the downregulation of c-jun N-terminal kinase (JNK) (88). These results agree with the results obtained on human squamous non-small cell lung cancer and cervical cancer, where cases with high expression of podoplanin were characterized by a lower incidence of nodal metastasis (89-93). However, in head and neck cancers high podoplanin expression in cancer cells was found to be a negative prognostic factor (94-100). Interestingly, in some tumors cell lines derived from these tumor types e.g., invasive ductal breast carcinoma, lung, pancreatic and colorectal adenocarcinoma, podoplanin expression in cancer cells is rarely observed $(27,28,36,37,53)$. There is abundant evidence pointing to elevated podoplanin expression in cancers. This shows squamous differentiation in comparison to those of the adenocarcinoma type (Fig. 2A and B) (42,52,53).

Podoplanin was also proposed to be a hallmark of tumor initiating cells in squamous cell cancers since podoplaninpositive cancer cells exerted augmented tumorigenic potential as compared to cells without podoplanin expression $(101,102)$. In normal epidermis, podoplanin expression is observed in the basal layer and rises in the precancerous lesions (actinic keratosis) and skin squamous cell cancer $(103,104)$. Moreover, the A431 squamous cancer cell line derived from the vulva and the TE-11 cell line derived from esophageal squamous cell cancer showed divergent 
subpopulations with respect to podoplanin expression. Cells expressing podoplanin could give rise to both podoplanin expressing and non-expressing cells. Upon subcutaneous injection into SCID mice, tumors bearing podoplanin expressing cells were characterized by augmented growth as compared to tumors generated by cells without podoplanin expression $(101,102)$. In addition, podoplanin-positive A431 cells exhibited high expression of CD44 and sonic hedgehog (SHH), which are both markers of tumor initiating cells (102).

\section{Podoplanin expression in cells of normal and cancerous stroma}

Skin cancer. Podoplanin expression was first studied as a potential marker of lymphangiogenesis. The assessment of this process in numerous tumors led to the discovery of podoplanin expression in cancer cells as well as 'activated fibroblasts' (42,52). At first, podoplanin expression, known as PA2.26, antigen was noted in the membrane of fibroblasts in vivo (NIH-3T3, Swiss-3T3 and 10T1/2) and in fibroblasts of tissue sections of skin exposed to wounding or stimulation with 12-O-tetradecanoylphorbol-13-acetate (TPA) (45). In this study, in normal, untreated skin podoplanin expression was not noted in the basal and suprabasal skin layers, suggesting that podoplanin may be a marker of cell activity in skin carcinogenesis (45).

Lung cancer. There is increasing evidence that podoplanin is possibly expressed in CAFs of cancerous stroma, which contribute to the progression of numerous tumors (Fig. 2C-F) $(4,7)$. To date, podoplanin-positive CAFs are best characterized in lung adenocarcinoma. Kawase et al identified podoplaninpositive CAFs in tissue sections of 54 out of 177 cases of lung adenocarcinoma and confirmed its expression on the protein level in CAFs isolated from some of the tumors (28). It is noteworthy that podoplanin expression in CAFs was higher in comparison to non-cancerous fibroblasts isolated from surrounding tissues of the same patients (28). In this study cohort, podoplanin expression in CAFs was associated with a history of smoking, a primary tumor that was larger in size, the presence of lymph node metastasis, advanced pathological stage, poor grade of differentiation, as well as vascular and pleural invasion. Moreover, patients whose tumors were characterized by podoplanin expression in CAFs had significantly shorter overall survival in comparison to patients whose CAFs did not exhibit podoplanin expression (28). Podoplanin expressing CAFs were also identified as a marker of poor prognosis in other studies conducted on lung adenocarcinoma $(35,105,106)$. Interestingly, podoplanin expression in CAFs had no prognostic significance in squamous lung cancer, which is characterized by elevated expression of podoplanin in cancer cells $(35,107)$. As in cancer cells, tumor promoting effects of podoplanin-positive CAFs were found to be mediated by elevated RhoA activity, similar to observations in other cell types $(54,80,81,108)$. Human fibroblasts isolated from vascular adventitia with ectopic expression of podoplanin exerted elevated RhoA activity. Injection of these cells was shown to augment tumor formation of human lung adenocarcinoma cell line A549 upon co-injection into SCID mice, as compared to A549 cells co-injected with control human fibroblasts (108).
As the A549 cell line does not express CLEC-2, the mechanism of enhanced tumor formation by podoplanin expressing fibroblasts remains to be elucidated in order to determine if this interaction is contact dependent or mediated via secretion of other stimulatory factors. In their previous study, the authors showed that fibroblasts derived from human vascular adventitia enhanced lung adenocarcinoma tumor formation in vitro and in vivo of lung adenocarcinoma cell lines (A549, PC-14, CRL-5807) (105). Moreover, the experiments revealed that the podoplanin expressing subset of these fibroblasts enhanced tumor formation and lymph node metastasis of A549 cells as compared to the subset lacking podoplanin expression (105). Interestingly, podoplanin expressing CAFs were also identified in nodal metastases of lung adenocarcinoma and were associated with poor overall survival of patients with pathological N2 stage III cancers. However, the presence of podoplanin expressing CAFs in the metastatic lesion did not correlate with any other clinicopathological factors of the patients (109). Recently, Ono et al showed that podoplanin assessment in CAFs of stage I human squamous cell carcinoma, in addition to other immunohistochemical markers, may identify patients with poor outcome risk (110). In this study, a combined survival analysis of patients with low E-cadherin expression in cancer cells and high podoplanin expression in CAFs revealed that only $7 \%$ of the patients achieved the 5-year overall survival time (110).

Breast cancer. Podoplanin expression in CAFs was also identified as a negative prognosis marker of invasive ductal breast carcinoma (IDC) $(27,36)$. In normal breast and mastopathies, podoplanin expression was noted in myoepithelial cells surrounding the duct cells $(27,36,104)$. Although, the percentage of cases showing podoplanin expression in the study of Pula et al (27) and Schoppmann et al (36) differed, podoplanin expressing CAFs were associated with nodal involvement, poor differentiation grade and negative estrogen receptor status. Of the analyzed invasive lobular breast cancers, only one out of 48 analyzed cases showed podoplanin immunoreactivity in CAFs (36). In both studies the authors analyzed the association between the podoplanin expression in CAFs and lymphatic vessel densities (LVD) in the intratumoral and peritumoral areas, but only in the study of Pula et al a significant rise of intratumoral LVD with increasing expression of podoplanin in CAFs was noted (27). This may indicate that podoplanin-positive CAFs can possibly be generated via EndEMT from LECs. This hypothesis remains to be clarified.

Intrahepatic cholangiocarcinoma. In a study conducted on 86 cases of intrahepatic cholangiocarcinoma, Aishima et al identified podoplanin-positive myofibroblasts in the tumor stroma of 33 cases. The presence of these cells was associated with lymph node metastasis and poor outcome of the patients (111).

Colorectal carcinoma. Although the majority of recently published studies identified podoplanin expression in CAFs as an unfavorable marker of prognosis, expression of podoplanin in the tumor stroma of colorectal cancer was shown to be associated with good outcome of the patients (37). Moreover, cases 
characterized by podoplanin expression in the tumor stroma had shallower depths of tumor invasion, were localized more distally and had a lower incidence of liver metastasis (37). These contradictory results were supported with in vitro tumor invasion assays, which showed that colon adenocarcinoma cell lines (HCT116 and HCT15) exerted augmented invasive potential, when co-cultured with fibroblasts with podoplaninsiRNA mediated knock-down (37).

Uterine cervical carcinoma. The prognostic value of podoplanin expression in CAFs was also studied in uterine cervical carcinoma. Similar to the results obtained in colorectal carcinoma, cases characterized by podoplanin expression in CAFs had lower incidences of lymph node metastases (112). Although the proportion of fatal cases in this group was smaller than in cases without podoplanin in CAFs, this trend was not statistically significant (112). The presence of podoplanin expression in the squamous cell carcinomas of the uterine cervix was also noted by Dumoff et al, who showed that its levels in the stroma of invasive tumors were significantly elevated in comparison to normal stroma of the cervix. However, the prognostic significance of this finding was not assessed in this study (91).

Adenocarcinoma of the esophagus. Podplanin expression in CAFs was recently shown in adenocarcinoma of the esophagus, whereas none of its expression was noted in the precursor lesions of this cancer (Barret's mucosa without dysplasia, with low grade and high grade dysplastic Barret's mucosa) (113). Twenty-two of the 200 (11\%) studied adenocarcinomas were identified as podoplanin expressing and these cases were characterized by an advanced tumor stage, more frequent lymph node involvement and lymphatic vessel involvement. In addition, podoplanin expression in CAFs was identified as an independent marker of poor prognosis in the analyzed adenocarcinoma patients cohort supporting its role in the progression of this cancer (113). Interestingly, only 3\% of the analyzed lymph node metastases revealed podoplanin expression.

Other cancers. A comprehensive study aimed at identifying podoplanin expression in the cancerous stroma of various cancers was undertaken by Kitano et al (35). The most abundant podoplanin expressing CAFs were found in colorectal, stomach and biliary tract and pancreatic cancer, whereas cancers of the bladder, lung, liver, uterine body, prostate and ovary were characterized by expression in less than half of the CAFs. In thyroid cancers no podoplanin expression in the cancerous stroma was noted (35). Although the authors reported that podoplanin expression in CAFs was associated with higher primary size, the presence of lymph node metastasis, advanced disease stage, higher LVD and lymphatic and blood vessel invasion, these results should be interpreted with caution since the statistical analysis was performed on a pooled cohort of the analyzed cancers (35).

\section{Conclusion}

Recent years have brought new insights into the biological role of podoplanin. It is becoming apparent, that this widely expressed protein may become a key target for future anticancer therapies, regardless of its expression in cancer as well as CAFs. Nevertheless, the diversity of the biological roles of podoplanin in normal and cancerous transformed tissues requires further studies in order to better understand cancerstromal interactions.

\section{Acknowledgements}

This study was part of the 'Wrovasc - Integrated Cardiovascular Centre' project, co-financed by the European Regional Development Fund, within the Innovative Economy Operational Program, 2007-2013.

\section{References}

1. Balkwill $\mathrm{F}$ and Mantovani A: Inflammation and cancer: back to Virchow? Lancet 357: 539-545, 2001.

2. Balkwill FR and Mantovani A: Cancer-related inflammation: common themes and therapeutic opportunities. Semin Cancer Biol 22: 33-40, 2012.

3. Ruffell B, Affara NI and Coussens LM: Differential macrophage programming in the tumor microenvironment. Trends Immunol 33: 119-126, 2012.

4. Kalluri R and Zeisberg M: Fibroblasts in cancer. Nat Rev Cancer 6: 392-401, 2006.

5. Parsonage G, Filer AD, Haworth O, et al: A stromal address code defined by fibroblasts. Trends Immunol 26: 150-156, 2005.

6. Tomasek JJ, Gabbiani G, Hinz B, Chaponnier C and Brown RA: Myofibroblasts and mechano-regulation of connective tissue remodelling. Nat Rev Mol Cell Biol 3: 349-363, 2002.

7. Allen M and Louise Jones J: Jekyll and Hyde: the role of the microenvironment on the progression of cancer. J Pathol 223: 162-176, 2011.

8. Simian M, Hirai Y, Navre M, Werb Z, Lochter A and Bissell MJ: The interplay of matrix metalloproteinases, morphogens and growth factors is necessary for branching of mammary epithelial cells. Development 128: 3117-3131, 2001.

9. Bucala R: Review series - inflammation and fibrosis. Fibrocytes and fibrosis. QJM 105: 505-508, 2012.

10. Hanahan D and Weinberg RA: Hallmarks of cancer: the next generation. Cell 144: 646-674, 2011.

11. Pietras K and Ostman A: Hallmarks of cancer: interactions with the tumor stroma. Exp Cell Res 316: 1324-1331, 2010.

12. Allinen M, Beroukhim R, Cai L, et al: Molecular characterization of the tumor microenvironment in breast cancer. Cancer Cell 6: 17-32, 2004.

13. Potenta S, Zeisberg E and Kalluri R: The role of endothelial-tomesenchymal transition in cancer progression. Br J Cancer 99: 1375-1379, 2008.

14. Zeisberg EM, Potenta S, Xie L, Zeisberg M and Kalluri R: Discovery of endothelial to mesenchymal transition as a source for carcinoma-associated fibroblasts. Cancer Res 67: 10123-10128, 2007.

15. Orimo A, Gupta PB, Sgroi DC, et al: Stromal fibroblasts present in invasive human breast carcinomas promote tumor growth and angiogenesis through elevated SDF-1/CXCL12 secretion. Cell 121: 335-348, 2005 .

16. Boire A, Covic L, Agarwal A, Jacques S, Sherifi S and Kuliopulos A: PAR1 is a matrix metalloprotease-1 receptor that promotes invasion and tumorigenesis of breast cancer cells. Cell 120: 303-313, 2005.

17. Sternlicht MD, Lochter A, Sympson CJ, et al: The stromal proteinase MMP3/stromelysin-1 promotes mammary carcinogenesis. Cell 98: 137-146, 1999.

18. Cheng N, Bhowmick NA, Chytil A, et al: Loss of TGF-beta type II receptor in fibroblasts promotes mammary carcinoma growth and invasion through upregulation of TGF-alpha-, MSP- and HGF-mediated signaling networks. Oncogene 24: 5053-5068, 2005.

19. Bhowmick NA, Chytil A, Plieth D, et al: TGF-beta signaling in fibroblasts modulates the oncogenic potential of adjacent epithelia. Science 303: 848-851, 2004. 
20. Duncan MR, Frazier KS, Abramson S, et al: Connective tissue growth factor mediates transforming growth factor beta-induced collagen synthesis: down-regulation by cAMP. FASEB J 13: 1774-1786, 1999.

21. Yang F, Tuxhorn JA, Ressler SJ, McAlhany SJ, Dang TD and Rowley DR: Stromal expression of connective tissue growth factor promotes angiogenesis and prostate cancer tumorigenesis. Cancer Res 65: 8887-8895, 2005.

22. Levental KR, Yu H, Kass L, et al: Matrix crosslinking forces tumor progression by enhancing integrin signaling. Cell 139 891-906, 2009.

23. Erez N, Truitt M, Olson P, Arron ST and Hanahan D: Cancerassociated fibroblasts are activated in incipient neoplasia to orchestrate tumor-promoting inflammation in an NF-kappaBdependent manner. Cancer Cell 17: 135-147, 2010.

24. Vermeulen L, De Sousa EMF, van der Heijden M, et al: Wnt activity defines colon cancer stem cells and is regulated by the microenvironment. Nat Cell Biol 12: 468-476, 2010.

25. de Sousa EM, Vermeulen L, Richel D and Medema JP: Targeting Wnt signaling in colon cancer stem cells. Clin Cancer Res 17: 647-653, 2011.

26. Chang HY, Chi JT, Dudoit S, et al: Diversity, topographic differentiation, and positional memory in human fibroblasts. Proc Nat Acad Sci USA 99: 12877-12882, 2002.

27. Pula B, Jethon A, Piotrowska A, et al: Podoplanin expression by cancer-associated fibroblasts predicts poor outcome in invasive ductal breast carcinoma. Histopathology 59: 1249-1260, 2011.

28. Kawase A, Ishii G, Nagai K, et al: Podoplanin expression by cancer associated fibroblasts predicts poor prognosis of lung adenocarcinoma. Int J Cancer 123: 1053-1059, 2008

29. Mork C, van Deurs B and Petersen OW: Regulation of vimentin expression in cultured human mammary epithelial cells Differentiation 43: 146-156, 1990.

30. Strutz F, Okada H, Lo CW, et al: Identification and characterization of a fibroblast marker: FSP1. J Cell Biol 130: 393-405, 1995.

31. Rettig WJ, Garin-Chesa P, Healey JH, et al: Regulation and heteromeric structure of the fibroblast activation protein in normal and transformed cells of mesenchymal and neuroectodermal origin. Cancer Res 53: 3327-3335, 1993.

32. Huber MA, Kraut N, Park JE, et al: Fibroblast activation protein differential expression and serine protease activity in reactive stromal fibroblasts of melanocytic skin tumors. J Invest Dermatol 120: 182-188, 2003

33. Lai D, Ma L and Wang F: Fibroblast activation protein regulates tumor-associated fibroblasts and epithelial ovarian cancer cells. Int J Oncol 41: 541-550, 2012.

34. Sugimoto H, Mundel TM, Kieran MW and Kalluri R: Identification of fibroblast heterogeneity in the tumor microenvironment. Cancer Biol Ther 5: 1640-1646, 2006.

35. Kitano H, Kageyama S, Hewitt SM, et al: Podoplanin expression in cancerous stroma induces lymphangiogenesis and predicts lymphatic spread and patient survival. Arch Pathol Lab Med 134: $1520-1527,2010$

36. Schoppmann SF, Berghoff A, Dinhof C, et al: Podoplaninexpressing cancer-associated fibroblasts are associated with poor prognosis in invasive breast cancer. Breast Cancer Res Treat: Nov 19, 2012 (Epub ahead of print)

37. Yamanashi T, Nakanishi Y, Fujii G, et al: Podoplanin expression identified in stromal fibroblasts as a favorable prognostic marker in patients with colorectal carcinoma. Oncology 77: 53-62, 2009.

38. Nose K, Saito $\mathrm{H}$ and Kuroki T: Isolation of a gene sequence induced later by tumor-promoting 12-O-tetradecanoylphorbol13-acetate in mouse osteoblastic cells (MC3T3-E1) and expressed constitutively in ras-transformed cells. Cell Growth Differ 1: 511-518, 1990

39. Wetterwald A,Hoffstetter W,Cecchini MG, et al: Characterization and cloning of the E11 antigen, a marker expressed by rat osteoblasts and osteocytes. Bone 18: 125-132, 1996.

40. Breiteneder-Geleff S, Matsui K, Soleiman A, et al: Podoplanin, novel 43-kd membrane protein of glomerular epithelial cells, is down-regulated in puromycin nephrosis. Am J Pathol 151: 1141-1152, 1997

41. Breiteneder-Geleff S, Soleiman A, Kowalski H, et al: Angiosarcomas express mixed endothelial phenotypes of blood and lymphatic capillaries: podoplanin as a specific marker for lymphatic endothelium. Am J Pathol 154: 385-394, 1999.

42. Raica M, Cimpean AM and Ribatti D: The role of podoplanin in tumor progression and metastasis. Anticancer Res 28: 2997-3006, 2008 .
43. Marks A, Sutherland DR, Bailey D, et al: Characterization and distribution of an oncofetal antigen (M2A antigen) expressed on testicular germ cell tumours. Br J Cancer 80: 569-578, 1999.

44. Farr AG, Berry ML, Kim A, Nelson AJ, Welch MP and Aruffo A Characterization and cloning of a novel glycoprotein expressed by stromal cells in T-dependent areas of peripheral lymphoid tissues. J Exp Med 176: 1477-1482, 1992.

45. Gandarillas A, Scholl FG, Benito N, Gamallo C and Quintanilla M: Induction of PA2.26, a cell-surface antigen expressed by active fibroblasts, in mouse epidermal keratinocytes during carcinogenesis. Mol Carcinog 20: 10-18, 1997.

46. Zimmer G, Oeffner F, Von Messling V, et al: Cloning and characterization of gp36, a human mucin-type glycoprotein preferentially expressed in vascular endothelium. Biochem J 341: 277-284, 1999.

47. Kato Y, Fujita N, Kunita A, et al: Molecular identification of Aggrus/T1alpha as a platelet aggregation-inducing factor expressed in colorectal tumors. J Biol Chem 278: 51599-51605, 2003.

48. Schacht V, Ramirez MI, Hong YK, et al: T1alpha/podoplanin deficiency disrupts normal lymphatic vasculature formation and causes lymphedema. EMBO J 22: 3546-3556, 2003.

49. Schacht V, Dadras SS, Johnson LA, Jackson DG, Hong Y-K and Detmar M: Up-regulation of the lymphatic marker podoplanin, a mucin-type transmembrane glycoprotein, in human squamous cell carcinomas and germ cell tumors. Am J Pathol 166: 913-921, 2005.

50. Sonne SB, Herlihy AS, Hoei-Hansen CE, et al: Identity of M2A (D2-40) antigen and gp36 (Aggrus, T1A-2, podoplanin) in human developing testis, testicular carcinoma in situ and germ-cell tumours. Virchows Arch 449: 200-206, 2006.

51. Kaneko MK, Kato Y, Kitano T and Osawa M: Conservation of a platelet activating domain of Aggrus/podoplanin as a platelet aggregation-inducing factor. Gene 378: 52-57, 2006.

52. Wicki A and Christofori G: The potential role of podoplanin in tumour invasion. Br J Cancer 96: 1-5, 2007.

53. Martin-Villar E, Yurrita MM, Fernandez-Munoz B, Quintanilla M and Renart J: Regulation of podoplanin/PA2.26 antigen expression in tumour cells. Involvement of calpainmediated proteolysis. Int J Biochem Cell Biol 41: 1421-1429, 2009.

54. Martin-Villar E, Megias D, Castel S, Yurrita MM, Vilaro S and Quintanilla M: Podoplanin binds ERM proteins to activate RhoA and promote epithelial-mesenchymal transition. J Cell Sci 119: 4541-4553, 2006

55. Kriehuber E, Breiteneder-Geleff $\mathrm{S}$, Groeger M, et al: Isolation and characterization of dermal lymphatic and blood endothelial cells reveal stable and functionally specialized cell lineages. J Exp Med 194: 797-808, 2001

56. Hwang YS, Xianglan Z, Park KK and Chung WY: Functional invadopodia formation through stabilization of the PDPN transcript by IMP-3 and cancer-stromal crosstalk for PDPN expression. Carcinogenesis 33: 2135-2146, 2012.

57. Honma M, Minami-Hori $M$, Takahashi $H$ and Iizuka $H$ : Podoplanin expression in wound and hyperproliferative psoriatic epidermis: regulation by TGF-beta and STAT-3 activating cytokines, IFN-gamma, IL-6, and IL-22. J Dermatol Sci 65: 134-140, 2012

58. Cortez MA, Nicoloso MS, Shimizu M, et al: miR-29b and miR-125a regulate podoplanin and suppress invasion in glioblastoma. Genes Chromosomes Cancer 49: 981-990, 2010.

59. Wicki A, Lehembre F, Wick N, Hantusch B, Kerjaschki D and Christofori G: Tumor invasion in the absence of epithelialmesenchymal transition: podoplanin-mediated remodeling of the actin cytoskeleton. Cancer Cell 9: 261-272, 2006.

60. Kunita A, Kashima TG, Ohazama A, Grigoriadis AE and Fukayama M: Podoplanin is regulated by AP-1 and promotes platelet aggregation and cell migration in osteosarcoma. Am J Pathol 179: 1041-1049, 2011.

61. Kreuger J, Nilsson I, Kerjaschki D, Petrova T, Alitalo K and Claesson-Welsh L: Early lymph vessel development from embryonic stem cells. Arterioscler Thromb Vasc Biol 26: 1073-1078, 2006

62. Petrova TV, Makinen T, Makela TP, et al: Lymphatic endothelial reprogramming of vascular endothelial cells by the Prox-1 homeobox transcription factor. EMBO J 21: 4593-4599, 2002.

63. Groger M, Loewe R, Holnthoner W, et al: IL-3 induces expression of lymphatic markers Prox-1 and podoplanin in human endothelial cells. J Immunol 173: 7161-7169, 2004. 
64. Ramirez MI, Millien G, Hinds A, Cao Y, Seldin DC and Williams MC: T1alpha, a lung type I cell differentiation gene, is required for normal lung cell proliferation and alveolus formation at birth. Dev Biol 256: 61-72, 2003.

65. Fu J, Gerhardt H, McDaniel JM, et al: Endothelial cell O-glycan deficiency causes blood/lymphatic misconnections and consequent fatty liver disease in mice. J Clin Invest 118: 3725-3737, 2008.

66. Uhrin P, Zaujec J, Breuss JM, et al: Novel function for blood platelets and podoplanin in developmental separation of blood and lymphatic circulation. Blood 115: 3997-4005, 2010

67. Suzuki-Inoue $\mathrm{K}$, Kato $\mathrm{Y}$, Inoue $\mathrm{O}$, et al: Involvement of the snake toxin receptor CLEC-2, in podoplanin-mediated platelet activation, by cancer cells. J Biol Chem 282: 25993-26001, 2007.

68. Kato Y, Kaneko MK, Kunita A, et al: Molecular analysis of the pathophysiological binding of the platelet aggregation-inducing factor podoplanin to the C-type lectin-like receptor CLEC-2 Cancer Sci 99: 54-61, 2008.

69. Ogasawara S, Kaneko MK, Price JE and Kato Y: Characterization of anti-podoplanin monoclonal antibodies: critical epitopes for neutralizing the interaction between podoplanin and CLEC-2. Hybridoma 27: 259-267, 2008.

70. Nieswandt B, Hafner M, Echtenacher B and Mannel DN: Lysis of tumor cells by natural killer cells in mice is impeded by platelets. Cancer Res 59: 1295-1300, 1999.

71. Katagiri Y, Hayashi Y, Baba I, Suzuki H, Tanoue K and Yamazaki $\mathrm{H}$ : Characterization of platelet aggregation induced by the human melanoma cell line HMV-I: roles of heparin, plasma adhesive proteins, and tumor cell membrane proteins. Cancer Res 51: 1286-1293, 1991.

72. Suzuki-Inoue K: Essential in vivo roles of the platelet activation receptor CLEC-2 in tumour metastasis, lymphangiogenesis and thrombus formation. J Biochem 150: 127-132, 2011.

73. Kaneko MK, Kunita A, Abe S, et al: A chimeric anti-podoplanin antibody suppresses tumor metastasis via neutralization and antibody-dependent cellular cytotoxicity. Cancer Sci 103: 1913-1919, 2012.

74. Suzuki-Inoue K, Inoue O, Ding G, et al: Essential in vivo roles of the C-type lectin receptor CLEC-2: embryonic/neonatal lethality of CLEC-2-deficient mice by blood/lymphatic misconnections and impaired thrombus formation of CLEC-2-deficient platelets. J Biol Chem 285: 24494-24507, 2010

75. May F, Hagedorn I, Pleines I, et al: CLEC-2 is an essential platelet-activating receptor in hemostasis and thrombosis. Blood 114: 3464-3472, 2009

76. Hughes CE, Navarro-Nunez L, Finney BA, Mourao-Sa D Pollitt AY and Watson SP: CLEC-2 is not required for platelet aggregation at arteriolar shear. J Thromb Haemost 8: 2328-2332, 2010.

77. Cueni LN, Chen L, Zhang H, et al: Podoplanin-Fc reduces lymphatic vessel formation in vitro and in vivo and causes disseminated intravascular coagulation when transgenically expressed in the skin. Blood 116: 4376-4384, 2010.

78. Lowe KL, Navarro-Nunez L and Watson SP: Platelet CLEC-2 and podoplanin in cancer metastasis. Thromb Res 129 (Suppl 1): S30-S37, 2012

79. Kalluri R and Weinberg RA: The basics of epithelial-mesenchymal transition. J Clin Invest 119: 1420-1428, 2009.

80. Scholl FG, Gamallo C and Quintanilla M: Ectopic expression of PA2.26 antigen in epidermal keratinocytes leads to destabilization of adherens junctions and malignant progression. Lab Invest 80: $1749-1759,2000$

81. Fernandez-Munoz B, Yurrita MM, Martin-Villar E, et al: The transmembrane domain of podoplanin is required for its association with lipid rafts and the induction of epithelialmesenchymal transition. Int J Biochem Cell Biol 43: 886-896, 2011.

82. Martin-Villar E, Fernandez-Munoz B, Parsons M, et al: Podoplanin associates with CD44 to promote directional cell migration. Mol Biol Cell 21: 4387-4399, 2010.

83. Oliferenko S, Paiha K, Harder T, et al: Analysis of CD44containing lipid rafts: recruitment of annexin II and stabilization by the actin cytoskeleton. J Cell Biol 146: 843-854, 1999.

84. Shen Y, Chen CS, Ichikawa H and Goldberg GS: SRC induces podoplanin expression to promote cell migration. J Biol Chem 285: 9649-9656, 2010.

85. Hogan C: Impact of interactions between normal and transformed epithelial cells and the relevance to cancer. Cell Mol Life Sci 69: 203-213, 2012
86. Acton SE, Astarita JL, Malhotra D, et al: Podoplanin-rich stromal networks induce dendritic cell motility via activation of the C-type lectin receptor CLEC-2. Immunity 37: 276-289, 2012.

87. Cueni LN, Hegyi I, Shin JW, et al: Tumor lymphangiogenesis and metastasis to lymph nodes induced by cancer cell expression of podoplanin. Am J Pathol 177: 1004-1016, 2010.

88. Suzuki H, Onimaru M, Yonemitsu Y, Maehara Y, Nakamura S and Sueishi K: Podoplanin in cancer cells is experimentally able to attenuate prolymphangiogenic and lymphogenous metastatic potentials of lung squamoid cancer cells. Mol Cancer 9: 287, 2010.

89. Suzuki H, Onimaru M, Koga T, et al: High podoplanin expression in cancer cells predicts lower incidence of nodal metastasis in patients with lung squamous cell carcinoma. Pathol Res Pract 207: 111-115, 2011.

90. Dumoff KL, Chu C, Xu X, Pasha T, Zhang PJ and Acs G: Low D2-40 immunoreactivity correlates with lymphatic invasion and nodal metastasis in early-stage squamous cell carcinoma of the uterine cervix. Mod Pathol 18: 97-104, 2005.

91. Dumoff KL, Chu CS, Harris EE, et al: Low podoplanin expression in pretreatment biopsy material predicts poor prognosis in advanced-stage squamous cell carcinoma of the uterine cervix treated by primary radiation. Mod Pathol 19: 708-716, 2006.

92. Ito $\mathrm{T}$, Ishii $\mathrm{G}$, Nagai $\mathrm{K}$, et al: Low podoplanin expression of tumor cells predicts poor prognosis in pathological stage IB squamous cell carcinoma of the lung, tissue microarray analysis of 136 patients using 24 antibodies. Lung Cancer 63: 418-424, 2009.

93. Shimada Y, Ishii G, Nagai K, et al: Expression of podoplanin, $\mathrm{CD} 44$, and p63 in squamous cell carcinoma of the lung. Cancer Sci 100: 2054-2059, 2009.

94. Yuan P, Temam S, El-Naggar A, et al: Overexpression of podoplanin in oral cancer and its association with poor clinical outcome. Cancer 107: 563-569, 2006.

95. Saigusa S, Mohri Y, Ohi M, et al: Podoplanin and SOX2 expression in esophageal squamous cell carcinoma after neoadjuvant chemo-radiotherapy. Oncol Rep 26: 1069-1074, 2011.

96. Kreppel M, Drebber U, Wedemeyer I, et al: Podoplanin expression predicts prognosis in patients with oral squamous cel carcinoma treated with neoadjuvant radiochemotherapy. Oral Oncol 47: 873-878, 2011.

97. Tateyama H, Sugiura H, Yamatani C and Yano M: Expression of podoplanin in thymoma: its correlation with tumor invasion, nodal metastasis, and poor clinical outcome. Hum Pathol 42: 533-540, 2011

98. Kreppel M, Scheer M, Drebber U, Ritter L and Zoller JE: Impact of podoplanin expression in oral squamous cell carcinoma: clinical and histopathologic correlations. Virchows Arch 456: 473-482, 2010.

99. Chuang WY, Yeh CJ, Wu YC, et al: Tumor cell expression of podoplanin correlates with nodal metastasis in esophageal squamous cell carcinoma. Histol Histopathol 24: 1021-1027, 2009.

100. Chao YK, Chuang WY, Yeh CJ, et al: Prognostic significance of high podoplanin expression after chemoradiotherapy in esophageal squamous cell carcinoma patients. J Surg Oncol 105: $183-188,2012$.

101. Rahadiani N, Ikeda J, Makino T, et al: Tumorigenic role of podoplanin in esophageal squamous-cell carcinoma. Ann Surg Oncol 17: 1311-1323, 2010

102. Atsumi N, Ishii G, Kojima M, Sanada M, Fujii S and Ochiai A: Podoplanin, a novel marker of tumor-initiating cells in human squamous cell carcinoma A431. Biochem Biophys Res Commun 373: 36-41, 2008

103. Toll A, Gimeno-Beltran J, Ferrandiz-Pulido C, et al: D2-40 immunohistochemical overexpression in cutaneous squamous cell carcinomas: a marker of metastatic risk. J Am Acad Dermatol 67: 1310-1318, 2012

104. Kalof AN and Cooper K: D2-40 immunohistochemistry - so far! Adv Anat Pathol 16: 62-64, 2009

105. Hoshino A, Ishii G, Ito T, et al: Podoplanin-positive fibroblasts enhance lung adenocarcinoma tumor formation: podoplanin in fibroblast functions for tumor progression. Cancer Res 71: 4769-4779, 2011.

106. Ito $M$, Ishii G, Nagai K, Maeda R, Nakano $Y$ and Ochiai A: Prognostic impact of cancer-associated stromal cells in stage I lung adenocarcinoma patients. Chest 142: 151-158, 2012

107. Kadota K, Huang CL, Liu D, et al: The clinical significance of the tumor cell D2-40 immunoreactivity in non-small cell lung cancer. Lung Cancer 70: 88-93, 2010. 
108. Ito S, Ishii G, Hoshino A, et al: Tumor promoting effect of podoplanin-positive fibroblasts is mediated by enhanced RhoA activity. Biochem Biophys Res Commun 422: 194-199, 2012.

109. Neri S, Ishii G, Taira T, et al: Recruitment of podoplanin positive cancer-associated fibroblasts in metastatic lymph nodes predicts poor prognosis in pathological N2 stage III lung adenocarcinoma. Ann Surg Oncol 19: 3953-3962, 2012

110. Ono S, Ishii G, Nagai K, et al: Podoplanin-positive cancer associated fibroblast could have prognostic value independent of cancer cell phenotype in stage I lung squamous cell carcinoma: utility of combining analysis of both cancer cell phenotype and cancer associated fibroblast phenotype. Chest: Oct 15, 2012 (Epub ahead of print). doi: 10.1378/chest.12-0913.
111. Aishima S, Nishihara Y, Iguchi T, et al: Lymphatic spread is related to VEGF-C expression and D2-40-positive myofibroblasts in intrahepatic cholangiocarcinoma. Mod Pathol 21: 256-264, 2008.

112. Carvalho FM, Zaganelli FL, Almeida BG, Goes JC, Baracat EC and Carvalho JP: Prognostic value of podoplanin expression in intratumoral stroma and neoplastic cells of uterine cervical carcinomas. Clinics 65: 1279-1283, 2010.

113. Schoppmann SF, Jesch B, Riegler MF, et al: Podoplanin expressing cancer associated fibroblasts are associated with unfavourable prognosis in adenocarcinoma of the esophagus. Clin Exp Metastasis 30: 441-446, 2013. 